\title{
POLYSILICON XYLOPHONE BAR MAGNETOMETERS
}

\author{
Dennis K. Wickenden, John L. Champion, R. Ben Givens, Thomas J. Kistenmacher, Jay L. Lamb, and Robert \\ Osiander \\ The Johns Hopkins University \\ Applied Physics Laboratory \\ Laurel, MD 20723
}

\begin{abstract}
The availability of miniature orientation or spin sensors capable of being mounted directly on a projectile would greatly simplify testing and evaluation procedures. It would also allow for the development of smart munitions by providing essential fuzing and guidance functions. One of the simplest orientation sensors is a magnetometer. The recently developed Johns Hopkins University Applied Physics Laboratory xylophone magnetometer is shown to be suitable for such applications. The xylophone magnetometer, based on a free-free resonating bar and utilizing the Lorentz force to measure vector magnetic fields, is intrinsically linear and has a wide dynamic range, measuring field strengths from nanoteslas to teslas. Furthermore, since its sensitivity, for a constant Lorentz force, is independent of size for resonating bars of the same material and aspect ratio, it is ideally suited for miniaturization. Various polysilicon xylophone bars have been designed, fabricated at the Cronos MUMPs foundry, and characterized. The collective output response has verified the size-independent scaling law and sensitivities of order $100 \mathrm{n}$ ' h have been achieved with drive currents as low as $20 \mu \mathrm{A}$. This drive current is limited by the sheet resistance of polysilicon and directly affects the sensitivity and dynamic range. Aspects of the various designs for polysilicon xylophone bar magnetometers are described, together with experimental results and projections for improving sensitivity and dynamic range.
\end{abstract}

\section{INTRODUCTION}

Typical techniques for measuring the orientation or spin rate of various types of munitions during the free flight phase of their test and evaluation include spin cards, high-speed video, spark range photographs, x-rays, and radar. All of these techniques are complex, expensive to implement, require special test ranges, and do not readily provide continuous recording [1]. The availability of miniature orientation sensors capable of being mounted directly on the munition would greatly simplify test and evaluation procedures and would also expand their application in munition systems. For example, they could serve two important functions. The first, as a compass, would measure the direction of travel of the munition with respect to the local Earth's magnetic field, and aid in its guidance. The second, as an angular ratc scnsor, would have fuzing applications. For example, the spin-up that occurs as the munition is fired could trigger an arming switch to activate a fuze. Alternatively, it could act as a timing device to air burst the munition after traveling a predetermined number of revolutions. A processor, in combination with the magnetometer, could be programmed to keep track of the number of revolutions the munition makes as it travels down range. If the munition's velocity is known, then the number of revolutions could be set to initiate the burst at the desired distance.
One of the simplest orientation or spin sensors is a magnetometer. In either application, the sensor measures the vector components of the Earth's magnetic field as a function of time. A multiple sensor device is needed to avoid nulls that will occur when the sensitive axis aligns with magnetic field lines. To date, the use of magnetometers to assist with the determination of a projectilc's motion or heading has becn severcly limited. Two obvious reasons are the low sensitivity of standard compasses and the size and weight constraints of standard magnetometers. The multi-sensor requirement leads naturally to the necessity of further miniaturization and microelectromechanical system (MEMS)based implementation. The goal of developing MEMS-based magnetometers has lead to the development of other new devices such as piezoresistive [2] and magnetostrictive [3] cantilevers, magnetoresistive films [4], and magnetometers based on electron tunneling effects [5]. While these methods can be used to create miniature magnetometers, their sensitivity has not reached the nanotesla range required for precision instruments. This article describes the development of MEMS-based devices based on the Johns Hopkins University Applied Physics Laboratory's xylophone bar magnetometer [6], with particular emphasis on their use in projectile applications.

\section{XYLOPHONE PRINCIPLES}

The resonating xylophone bar magnetometer uses the Lorentz force as a measure of the magnetic field vector. It offers the potential of being lightweight and operating in a low power mode. True to its name, the device is based on a classical resonating xylophone bar. The principle of operation is shown in Figure 1.

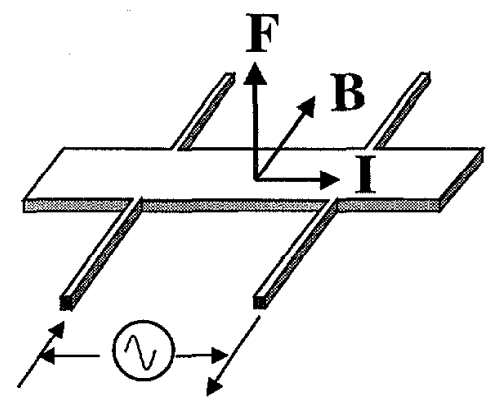

Figure 1 Principle of operation of xylophone bar magnetometer

The active element is a conductive bar supported at the nodes of its fundamental mode, of frequency $f_{o}$, of mechanical vibration. An alternating sinusoidal current $[I \cos (2 \pi f t)]$ is fed along the bar through the supports. In the absence of a magnetic field the bar will remain stationary. 
In the presence of a magnetic field, $B$, the Lorentz force $F$, normal to the bar and the drive current, is given by:

$F=I \times B \cos (2 \pi f t)$

This force causes the xylophone bar to vibrate, with a displacement, $d$, at the mid-point of the bar being given by:

$$
d=\frac{d_{d c}}{\sqrt{\left[1-\left(\frac{f}{f_{0}}\right)^{2}\right]^{2}+\left(\frac{f}{Q f_{0}}\right)^{2}}}
$$

The static deflection of a beam, of length $l$, width $a$, and thickness $b$, simply supported at both ends due to the Lorentz force uniformly distributed along its length is given by [7]:

$$
d_{d c}=\frac{5 F l_{2}^{4}}{384 E I_{B}}
$$

where $I_{B}=$ area moment of inertia $=a b^{3} / 12, \mathrm{E}=$ Young's modulus, and $l_{2}$ is the distance between the supports.

The phase angle between the driving current and the deflection is given by:

$$
\phi=\arctan \left(\frac{f \cdot f_{0}}{Q\left(f_{0}^{2}-f^{2}\right)}\right)
$$

In the case of an ideal free-free bar, where the effect of the supports can be neglected, the resonance frequency of the fundamental mode of the bar is given by:

$$
f_{0}=\frac{22.4}{2 \pi} \sqrt{\frac{E I_{B}}{\rho a b l^{4}}}
$$

It can be seen from these equations that at resonance $\left(f=f_{o}\right)$, the deflection of the xylophone bar is linearly proportional to the amplitude of the drive current, $I$, the mechanical $Q$ of the structure, and the magnetic flux density, $B$. Furthermore, since the xylophone bar includes no magnetic material, the sensor does not saturate. This results in a magnetometer with an extremely wide linear dynamic range. Macro-devices, chemically milled form $\mathrm{Cu}$ Be foils, with dimensions of $5 \mathrm{~mm} \times 0.5 \mathrm{~mm} \times 0.025 \mathrm{~mm}, \mathrm{Q}$ factors in the range $1000-5000$, and drive currents of the order of one amp, have demonstrated dynamic ranges in excess of $80 \mathrm{~dB}$ and a noise floor below $0.1 \mathrm{nT} / \mathrm{V} \mathrm{Hz}$ [8].

\section{RESULTS}

The xylophone bar magnetometer design readily lends itself to miniaturization, and importantly, as equation (3) shows, that a scaling of all dimensions (length, thickness, and width) does not change the sensitivity (deflection) of the xylophone bar for a constant Lorentz force. Several generations of MEMS- processed polysilicon magnetometers have been designed and fabricated using Multi-User MEMS Processes (MUMPs) foundry service at MCNC. A schematic diagram of a typical device is shown in Figure 2. The lower electrode consists of a $0.5-\mu \mathrm{m}$ thick

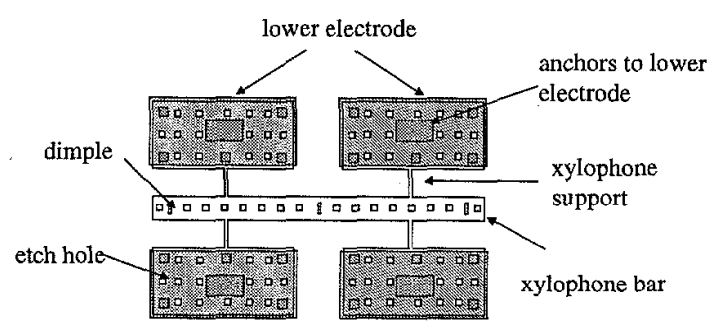

Figure 2 A schematic diagram of a typical device

polysilicon layer patterned on the silicon nitride-coated silicon substrate. The xylophone bar, support electrodes, and mounting pads are fabricated from $2 \mu \mathrm{m}$ thick polysilicon suspended (after release of the sacrificial silicon dioxide) $2 \mu \mathrm{m}$ above the nitride layer or lower electrode. The mounting pads are attached to the lower electrode by indicated patterns to provide a rigid anchor that minimizes vibrational coupling with the xylophone bar [9]. Contact between the xylophone bar and the nitride layer is minimized by having $0.75 \mu \mathrm{m}$ deep "dimples" in the center and toward the ends of the xylophone bar. Lastly, etch holes are incorporated to facilitate a clean release of the completed structure. An SEM micrograph of a packaged $500 \times 50-\mu \mathrm{m}$ xylophone bar is presented in Figure 3. The micrograph clearly displays the anchor pattern, the support arms, the mounting pads, and the topographical evidence of the dimples. It also demonstrates the clean release of the xylophone structure.

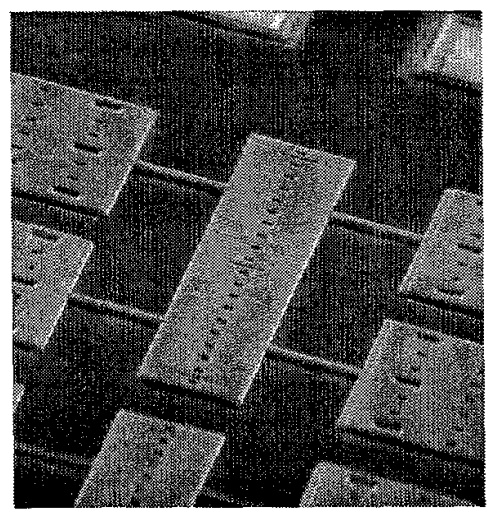

Figure 3 SEM micrograph of a packaged $500 \times 50$ - $\mu$ m xylophone bar

Evaluation of these polysilicon devices has been undertaken in a vacuum test chamber using an optical beam deflection setup. All devices tested to date have worked very well in static magnetic fields and exhibit mechanical $Q$-factors in excess of 20,000 at reduced pressures of $15-20$ mTorr.

Figure 4 illustrates results obtained by scanning the frequency of the drive current through a $500 \times 50 \mu \mathrm{m}$ xylophone bar with 45 $\mu \mathrm{m}$ long $\times 4 \mu \mathrm{m}$ wide supports operating in a static magnetic field. The rms current through the device was $22 \mu \mathrm{A}$, the magnetic flux 
density was on the order of $\mathrm{mT}$ (from a nearby permanent magnet) and the test chamber pressure was 35 mTorr. From this data, the resonance frequency was found to be $78.15 \mathrm{kHz}$. The predicted frequency from equation (5) is $68.4 \mathrm{kHz}$.

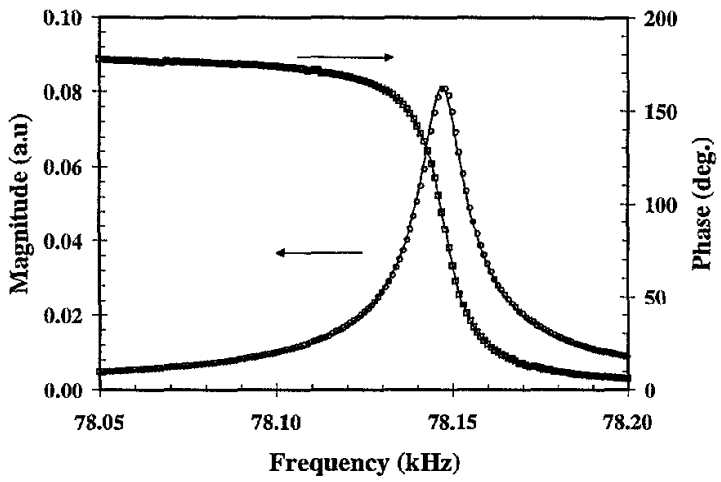

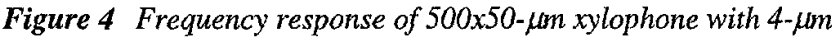
wide support arms

The difference between the observed and predicted resonance frequency was attributed to the increased torsional stiffness of the xylophone support arms. This was confirmed by a detailed analysis of the mechanical properties of the structures. In this analysis, each of the support arms was modeled as a combination of vertical and torsional springs ( $K_{v}$ and $K_{r}$, respectively) and a concentrated mass $(M)$; and, the resonator was modeled as a beam using Bernoulli-Euler theory. Use of this theory, rather than the more complex Timoshenko theory, was justified because the wavelength of vibration is much larger than the thickness of the beam. The differential equation governing the motion of a Bernoulli-Euler beam undergoing harmonic motion $w(x, t)$ at an angular frequency $\omega$ is given by:

$$
E I_{B} w^{i v}+\omega^{2} \rho A w=0
$$

The effects of the two springs and concentrated mass enter into the expressions for the boundary conditions. Here, the boundaries include both ends of the beam as well as the support points. The zero-moment and zero-shear boundary conditions are used at both of the free ends. The motion of the beam is restrained to some degree in both displacement and rotation by the two elastic springs. The concentrated mass also adds inertia to the beam. Because the springs and mass are attached to the nodal points, neither $K_{v}$ nor the mass $M$ has any influence on the first bending mode of vibration. Only the torsional spring $K_{T}$ has an influence on this mode and results in the observed functional dependence of resonance frequency on support arm width, as illustrated in Figure 5. In this figure the solid line denotes the results of the analysis and the open circles represent the experimental results from $500 \times 50 \times 2 \mu \mathrm{m}$ xylophone bars with different support arm widths. The agreement is excellent and well within the manufacturing tolerances of the MEMS processing.

A plot of the output response of the polysilicon xylophone magnetometer with $4 \mu \mathrm{m}$ support arm widths as a function of impressed magnetic flux density up to $150 \mu \mathrm{T}$ is shown in Figure 6. The superimposed line is a least-squares fit to the data, and it is apparent that the magnetometer response is linear over the range of magnetic flux density shown. An estimate of the ultimate noise

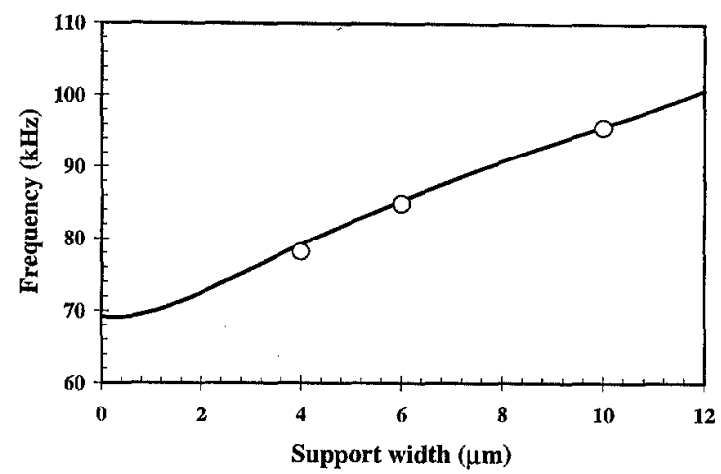

Figure 5 Resonance frequency as a function of support arm width

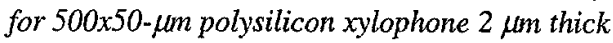

floor of the xylophone magnetometer, based on thermomechanical noise (Brownian motion due to the Langevin force) and, to a lesser extent, Johnson noise (current noise that couples with the magnetic field), yields a value of the order of $100 \mathrm{p} T \bullet \mathrm{A} / \sqrt{\mathrm{Hz}}$. This estimate calls out the very important role that the amplitude of the drive current plays in determining the ultimate field sensitivity of a xylophone bar magnetometer.

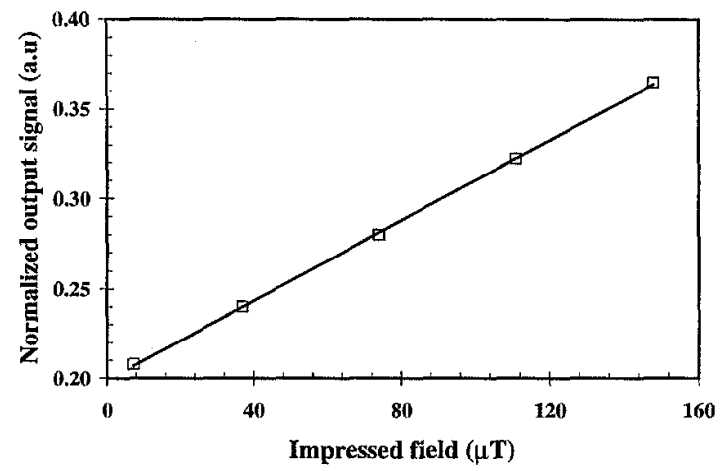

Figure 6. Output response of $500 \times 50-\mu \mathrm{m}$ xylophone magnetometer as a function of impressed magnetic flux density.

The length and width dimensions of the polysilicon xylophone bar magnetometer presented here are proportionally scaled from the $\mathrm{Cu}-\mathrm{Be}$ devices described above, while the thickness is an order of magnitude smaller than for direct scaling. The decreased sensitivity is identified as being a direct result of the high sheet resistivity of the polysilicon material (10 $\Omega /$ square) and its lower current carrying capability.

Current work is concentrated on reducing the resistance of the polysilicon devices. Approaches being adopted include feeding the drive current to the magnetometer from both sides of the xylophone bar, giving an immediate doubling of the drive current capability and improving the thermal symmetry of the device. In addition, magnetometers have been manufactured from $3.5-\mu \mathrm{m}$ thick poly1/poly2 layers, and the use of gold stripes deposited on various parts of the xylophone has been examined. An SEM micrograph of a released and packaged poly 2 devices with gold deposited on the electrodes, the support arms, and as stripes along the xylophone bar between the support arms, is reproduced in Figure 7. Poly1/poly2 $500 \times 50 \times 3.5-\mu \mathrm{m}$ bars with gold deposited on only the electrodes have resistances of approximately $150 \Omega$, resonance frequencies of $165 \mathrm{kHz}$ and Q-factors of between 30,000 and 40,000 at low 


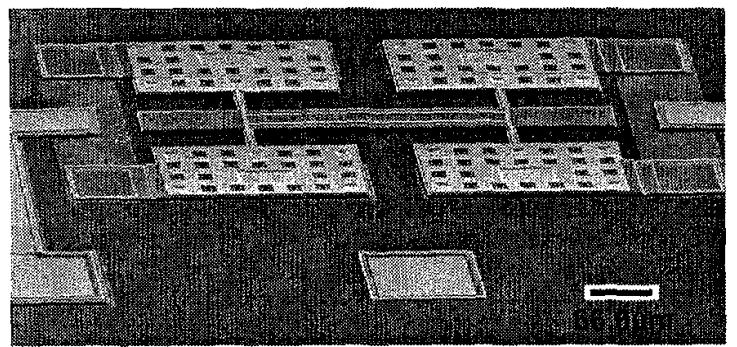

Figure 7. SEM micrograph of xylophone bar with the drive current fed from both sides of the xylophone and with gold deposited on the electrodes, the support arms, and along the bar.

pressures. The same bars with full gold strips on the support arms have resistances of approximately $80 \Omega$, increased resonance frequencies of $168 \mathrm{kHz}$, due to the increased stiffness of the support arms, and Q-factors of 10,000 . The bars with the gold strips along the xylophone bar have resistances of approximately $40 \Omega$, decreased resonance frequencies of approximately $164 \mathrm{kHz}$, due either to mass loading of the bar or to the decreased effective Young's modulus, and Q-factors in excess of 10,000 . Investigations are continuing in order to determine whether the drop in Q-factor observed when gold is deposited on the support arms or along the bar is a real effect or not.

\section{CONCLUSIONS}

Polysilicon devices based on the Johns Hopkins University Applied Physics Laboratory's xylophone bar magnetometer have been shown to be suitable for use as sensitive orientation or spin sensors for projectile applications. Such sensors will not only greatly simplify the test and evaluation of projectiles undergoing current development but could also lead to the appearance of smarter future munitions. $500 \times 50 \times 2-\mu \mathrm{m}$ devices have confirmed the scaling laws expected for xylophone bar magnetometers and have a predicted ultimate noise floor of the order of 100 $\mathrm{pT} \bullet \mathrm{A} / \mathrm{JHz}$. The drive current capable of being passed by the polysilicon limits the sensitivity. The drive current can be increased by the use of gold overlays but early indications are that these techniques may degrade the mechanical Q-factors.

\section{ACKNOWLEDGEMENTS}

We also acknowledge the support for this work by the U.S. Department of the Navy under Contract N00039-91-5301 and the U.S. Air Force Officc of Scicntific Research under Contract F49620-98-1-0500. The views and conclusions contained herein are those of the authors and should not be interpreted as necessarily representing the official policies or endorsements, either expressed or implied, of the U. S. Department of the Navy, the Air Force Office of Scientific Research, or the U. S. Government.

\section{REFERENCES}

1. B. S. Davis, T. Harkins, and L. Burke, "Flight test results of miniature, low-cost, spin, accelerometer, and yaw sensors," AIAA 35th Aerospace Sciences Meeting and Exhibit, AIAA 97-0422, Reno, NV, 1997.
2. C. Rossel, P. Bauer, D. Zech, J. Hoder, M. Willemin, and H. Keller, "Active microlevers as miniature torque magnctomcters," $J$. Appl. Phys, , 79, 8166-8173 (1996).

3. R. Osiander, S. A. Ecelberger, R. B. Givens, D. K. Wickenden, J. C. Murphy, and T.J. Kistenmacher, "A microelectromechanicalbased magnetostrictive magnetometer," Appl. Phys. Lett., 69, 29302931 (1996).

4. F. Y. Yang, K. Liu, K. Hong, D. H. Reich, P. C. Searson, and C. L. Chien, "Large magnetoresistance and field sensing characteristics of electro-deposited single-crystal bismuth thin films," Science 284, 1335-1337 (1999).

5. L. M. Miller, J. A. Podosek, E. Kruglick, T. W. Kenny, J. A. Kovacic, and W. J. Kaiser, "A $\mu$-magnetometer based on electron tunneling," Proc. IEEE Workshop on Micro Electro Mechanical Systems, (IEEE, New York) pp. 467-472 (1996).

6. R. B. Givens, J. C. Murphy, R. Osiander, T. J. Kistenmacher, and D. K. Wickenden, "A high sensitivity, wide dynamic range magnetometer designed on a xylophone resonator," Appl. Phys. Lett., 69, 2755-2757 (1996).

7. W. C. Young, Roark's Formulas for Stress and Strain, $6^{\text {ti }}$ Edition, McGraw-Hill, Ncw York, 1989.

8. R. B. Givens, D. K. Wickenden, D. A. Oursler, R. Osiander, . L. Champion, and T. J. Kistenmacher, "Heterodyne detection of alternating magnetic fields with a resonating xylophone bar magnetometer," Appl. Phys. Lett., 74, 1472-1474 (1999).

9. D. Sherman, "An Investigation of MEMS Anchor Design for Optimal Stiffness and Damping," Master's Project, University of California, Berkeley, May 1996. 